\title{
Atem. Künste, Technologien und Architekturen der Moderne und Gegenwart
}

Die Luft, die wir ein- und ausatmen wird nur höchst selten sichtbar. Doch nicht nur sie, auch die Atmung selbst vollzieht sich unauffällig. Sie bleibt zumeist unmerklich, leise und unbewusst. Die Luft wird erst als Atem augenscheinlich, wenn sie etwa in der Ausatmung mit sichtbaren Partikeln angereichert ist, in kalter Luft an einer Scheibe kondensiert oder in ein formbares Material eingeblasen wird.

Kultur- und Kunstgeschichten der Luft und des Atmosphärischen wurden schon vielfach geschrieben und haben ebenso wie naturund populärwissenschaftliche Publikationen über Atem und Atmung zum Erscheinungszeitpunkt dieses Bandes Konjunktur. ${ }^{1}$ Von Interesse ist dabei zumeist die Luft als Territorium sowie als leitendes Medium für Informationsfluss und Mobilität überhaupt. ${ }^{2}$ Die Praxis des Atmens wird weitaus seltener in den Fokus genommen und wenn doch, stehen eher diskursive Aspekte im Vordergrund. Lenart Škof und Petri Berndtson versammeln beispielsweise in Atmospheres of Breathing (2018) Beiträge zu historischen und aktuellen Philosophien des Atmens, darunter Luce Irigarays The Forgetting of Air in Heidegger (1999). ${ }^{3}$ Wie viele der aktuellen Atempublikationen referieren die Autor*innen zwar punktuell auf die Kunst- und Bildgeschichte. Im Fokus stehen jedoch hier wie andernorts die Literatur ${ }^{4}$ - mitunter erweitert um Theater und Musik. ${ }^{5}$

1 Zur Kulturgeschichte siehe exemplarisch: Peter Adey, Air: Nature and Culture, London 2014; Everard Mark, Breathing Space: The Natural and Unnatural History of Air, London 2015; Steven Connor, The Matter of Air: Science and the Art of the Ethereal, London 2010; Lothar Binger, Himmel, Rauch und Care-Pakete: Eine Geschichte der Luft, Berlin 1998.

2 Aspekte der Datenübertragung ebenso wie der Schwerkraft und der Schwerelosigkeit stehen dabei häufig im Fokus; ebenso Vorstellungen des Luftraumes als Äther. Siehe exemplarisch Albert Kümmel-Schnur/Jens Schröter (Hg.), Äther. Ein Medium der Moderne. Bielefeld 2008; Annick Bureaud, „What if...art and weightlessness“, in: Monika Bakke (Hg.), Going Aerial: Air, Art, Architecture, Maastricht 2006, S. 138-145.
3 Lenart Škof/Petri Berndtson (Hg.), Atmospheres of Breathing, New York 2018.

4 Dies gilt etwa für Rebecca Schönsee, Kunst und Pneuma: Von Hofmannsthals Lyrik des Hauchs zur Atemperformance, Wien 2013 und Lisa Siraganian, Modernism's Other Work: The Art Object's Political Life, Oxford 2012.

5 Für die zeitgenössische Performance-Kunst, die körperliche Präsenz und verbale Artikulationen häufig vereint, sind diese Untersuchungen somit äußerst anschlussfähig. Vgl. Havi Carel, „The Uses and Abuses of Air", in: Air. Visualising the Invisible in British Art, 1768-2017, Ausst.Kat. Royal Academy of Arts, Bristol 2017, S. 47-53. 
Zwar wurden in den letzten Jahren Atem und Atmung als Motiv, Substanz oder Technik in Kunst und Kultur erforscht. ${ }^{6}$ Jedoch liegt das Hauptinteresse der meisten Untersuchungen auf den spirituellen und religiösen Dimensionen und einem sehr breiten Begriff von Atmung: Pneuma und das Pneumatische oder spirituelle Kraftkonzepte wie Prana und Chi aus ostasiatischen Traditionen stehen dabei häufig im Vordergrund. ${ }^{7}$ Der damit einhergehende, vornehmlich sprachlich-konzeptuelle Zugriff fällt immer wieder auf, wenn Etymologien und Redensarten am Beginn von Auseinandersetzungen stehen und prägnant in diese eingebunden sind. ${ }^{8}$ Dieser methodische Zugriff kulminiert zumeist in allgemein gehaltenen Untersuchungen zu Wind, Luft, Hauch, Inspiration, Seele, Schöpfung und Leere. ${ }^{9}$

Der vorliegende Band hat dagegen zum Ziel, ästhetische, gestalterische, technologische und politische Phänomene und Praktiken konkret entlang des physiologischen Phänomens der Atmung zu untersuchen und kulturhistorisch einzuordnen. Als physiologischer Vorgang tritt der Atem etwa in olfaktorischen Kunstwerken in Erscheinung. Einher geht die Zunahme der Arbeit mit Düften seit geraumer Zeit mit dem kulturwissenschaftlichen Diskurs über den Geruchssinn als vermeintlich niedrigster, archaistischer ${ }^{10}$ und in der Forschung vernachlässigter Sinn. ${ }^{11}$ Auch ein gesteigertes Forschungsinteresse an Phänomenen der Synästhesie hat eine historische Beschäftigung mit Gerüchen und somit der Atmung stärker zutage gefördert. ${ }^{12}$

Doch im Olfaktorischen erschöpfen sich die künstlerischen und gestalterischen Bezüge zum Atem keineswegs. Dieser Band verfolgt

6 Vlad lonescu, Pneumatology: An Inquiry into the Representation of Wind, Air and Breath, Brüssel 2017; zu Mittelalter und Früher Neuzeit siehe Barbara Baert, Pneuma and the Visual Medium in the Middle Ages and Early Modernity, Leuven/Paris/Bristol 2016. Weiterhin siehe die zahlreichen Atem-bezogenen Arbeiten im Ausst.Kat. Bristol 2017 (wie Anm. 5) sowie in Bakke 2006 (wie Anm. 2) und Davina Quinlivan, The Place of Breath in Cinema, Edinburgh 2012, S. 13-17.

7 Ulli Seegers, Alchemie des Sehens. Hermetische Kunst im 20. Jahrhundert. Antonin Artaud, Yves Klein, Sigmar Polke, Köln 2003; lonescu 2017 (wie Anm. 6), Schönsee 2013 (wie Anm. 4).

8 Siehe exemplarisch Ziad Mahayni, Feuer, Wasser, Erde, Luft: Eine Phänomenologie der Natur am Beispiel der vier Elemente, Rostock 2003, S. 237f.; lonescu 2017 (wie Anm. 6), S. 9.

9 Marie-Amélie zu Salm-Salm, „Die Bedeutung des Atems als Thema und Material im Werk von Jeppe Hein und im kunsthistorischen Kontext", in: Jeppe Hein. Einatmen Innehalten - Ausatmen, Ausst.-Kat. Kunstmuseum Thun, Berlin 2018, S. 43-50, hier: S. 49; Ionescu 2017 (wie Anm. 6), S. 23; Seegers 2003 (wie Anm. 7); weiterführend siehe Georgios T. Halkias, „Between Breaths“, in: Bakke 2006 (wie Anm. 2), S. 48-61.
10 Roland Wetzel/Annja Müller-Alsbach, „Einleitung und Rückblick auf die Ausstellung Belle Haleine - Duft in der Kunst“, in: Belle Haleine. Der Duft in der Kunst. Interdisziplinäres Symposium, hrsg. v. Museum Tinguely Basel 2015, S. 7-18.

11 Siehe Gespräch zwischen Dorothée King und Matt Morris in diesem Band. Weiterführend zu Kunst und Design mit Gerüchen: Gwen-Aël Lynn/Debra Parr, Olfactory Art and The Political in an Age of Resistance, London 2021 [im Erscheinen]; Victoria Henshaw et al. (Hg.), Designing with Smell: Practices, Techniques and Challenges, London 2017; Ausst.-Kat. Basel 2015 (wie Anm. 10); Jim Drobnick, The Smell Culture Reader, New York 2006.

12 Literatur mit Bezügen zur Olfaktorik: Francesca Bacci/ David Melcher (Hg.), Art and the Senses, Oxford 2013; Robin Curtis/Marc Glöde/Gertrud Koch (Hg.), Synästhesie-Effekte. Zur Intermodalität der ästhetischen Wahrnehmung, München 2010; Mădălina Diaconu, Tasten, Riechen, Schmecken. Eine Ästhetik der anästhesierten Sinne, Würzburg 2005; Hans Adler/Ulrike Zeuch (Hg.), Synästhesie. Interferenz - Transfer - Synthese der Sinne, Würzburg 2002; Der Sinne der Sinne, Ausst-Kat. Kunst- und Ausstellungshalle der Bundesrepublik Deutschland, Bonn 1998. 
daher das Ziel, weitere Dimensionen ästhetischer Auseinandersetzungen mit dem Atem zu erschließen. Die Beiträge eruieren jeweils historisch spezifisch ausgehend von Bildern, Objekten, Filmen, Installationskunst, künstlerischen, experimentellen und handwerklichen Techniken sowie anhand von architektonischen Ideen und Praktiken die Bedeutung von Atem bzw. Atmung für gestalterische Prozesse, Bildfindungen sowie für ästhetische, körperliche und geistige Erfahrungen. Somit werden inhaltliche und methodische Ergänzungen und Neuperspektivierungen der bisherigen Forschung unternommen: Formen der Visualisierung des Atems, Fragen der Materialität, der Wahrnehmung sowie der Erzeugung konkreter Objekte und Räume, inklusive Techniken und Technologien der Herstellung, rücken in den Vordergrund. Diese vielfältigen Praktiken werden auf ihr gestalterisches Potential, ebenso wie auf ihre sozialen, politischen und ökologischen Dimensionen hin untersucht. Der Fokus auf das 20. und 21. Jahrhundert markiert dabei eben jene Epoche, in welcher die Atemluft wie nie zuvor durch Technologien gemessen, manipuliert, ja, gestaltet und mobilisiert wurde und wird. Da diese kulturhistorischen Entwicklungen maßgeblich auf dem 18. und 19. Jahrhundert fußen, beginnt diese Einleitung in die kulturund diskurshistorische Bild- und Gestaltungsgeschichte des Atems mit einem Exkurs in die Wissenschaftskultur vor 1900 und die frühe Industrialisierung.

\section{Atem, Kunst und visuelle Kultur: \\ Eine historische Spurensuche}

Das Interesse von Künstler*innen an Lufterscheinungen entfaltete sich im ausgehenden 18. sowie im 19. Jahrhundert vornehmlich entlang der Atmosphäre als ästhetisches Stimmungs-, Licht- und Wetterphänomen und damit entlang von Ästhetiken, die auf Traditionen der Frühen Neuzeit zurückgehen. ${ }^{13}$ Nun waren es jedoch die Atmosphären der Industrialisierung, welche insbesondere englische Künstler ${ }^{14}$ ins Bild setzten. Die rauchenden Schlote und verdunkelten Himmel galten häufig als Zeichen von Fortschritt, Produktivität, Wohlstand, als Sinnbild der menschgemachten Natur sowie als pittoreskes Motiv. ${ }^{15}$

13 Siehe exemplarisch Christina Storch, Wetter, Wolken und Affekte: Die Atmosphäre in der Malerei der Frühen Neuzeit, Berlin 2015.

14 Heute sind vornehmlich männliche Künstler bekannt.
15 Vgl. Sabine Beneke/Hans Ottomeyer (Hg.), Die zweite Schöpfung. Bilder der industriellen Welt vom 18. Jahrhundert bis in die Gegenwart, Ausst.-Kat. Martin-GropiusBau, Berlin 2002; Monika Wagner, Die Industrielandschaft in der englischen Malerei und Grafik 1770-1830, Frankfurt am Main 1979. Siehe außerdem Anna Scouter, „Dirty pretty things: Air pollution in art from JMW Turner to today", in: The Guardian.com, 28.10.2020, verfügbar unter: https://www.theguardian.com/artanddesign/2020/ oct/28/jmw-turner-air-pollution-in-art-rain-steam-andspeed (letzter Zugriff: 10.11.2020). 


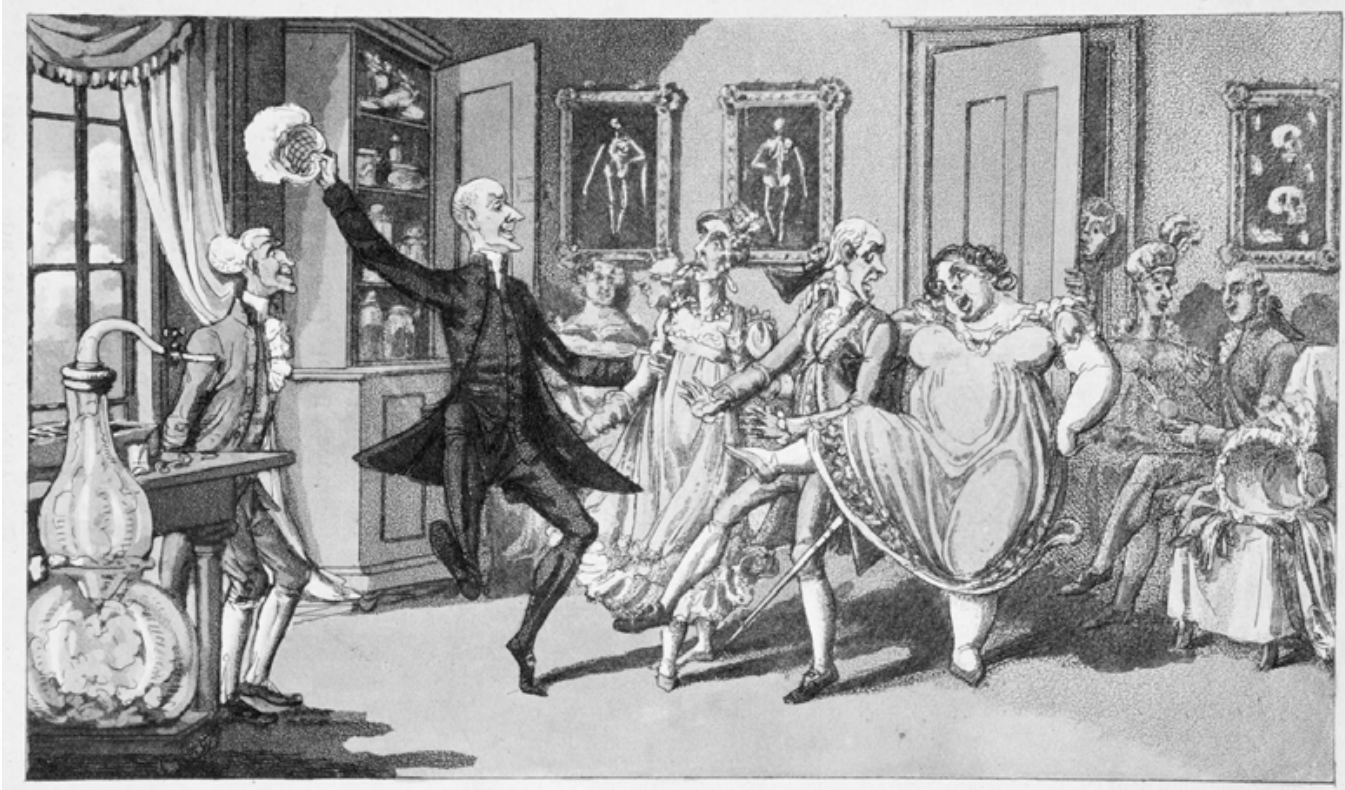


Sie fungierten seltener bzw. erst später als Mahnung angesichts zunehmender Gesundheitsgefährdung und Umweltzerstörung. ${ }^{16}$

Der Atem als solcher wurde in der modernen Landschaftsmalerei zunächst nicht direkt thematisch. In deren Zentrum stand die Atmosphäre als Anschauungsgegenstand. Anders verhielt es sich mit den Gattungen der Illustration, Karikatur und des Genrebildes, die häufig der visuellen Auseinandersetzung mit Luft- und Vakuumexperimenten dienten. Diese Bildtypen kursierten insbesondere in der ersten Hälfte des 19. Jahrhunderts. Künstler und Gestalter beschäftigten sich mit den naturwissenschaftlichen Forschungen zu Vakuum und Luftdruck seit der Mitte des 17. Jahrhunderts ${ }^{17}$ sowie der Erforschung von Luft als Gasgemisch ab den 1770er Jahren. ${ }^{18}$ Während Joseph Wright of Derbys berühmtes Gemälde An Experiment on a Bird in the Air Pump (1768) eine popularisierte Experimentalpraxis zum tödlichen Vakuum verhandelt, ${ }^{19}$ ironisieren satirische Bilder um 1820 damals zeitgemäße Experimente mit Lach- und anderen Gasen (Abb. 1). ${ }^{20}$

Zugleich war der Viktorianismus von einem wahrhaften „cult of ventilation and fresh air“ sowie einem ,intense fetishism of the breath“ geprägt. ${ }^{21}$ Im 19. Jahrhundert wurden in Europa im medizinischen Bereich Sauerstofftherapien und Beatmungstechnologien entwickelt, die bis heute medizinisch Anwendung finden. ${ }^{22}$ Atemobsessionen lassen sich auch in anderen Industrienationen feststellen. Der US-amerikanische Künstler George Catlin erforschte auf seinen Reisen die Atmung der native Americans. 1862 veröffentlichte er die Schrift The Breath of Life or mal-Respiration and its effects upon the enjoyments \& life of man, später neu aufgelegt als Shut Your Mouth and Save Your Life. Catlin führt darin zahlreiche physische und mentale Zivilisationskrankheiten auf die ,unnatürliche' Praxis der Mundatmung zurück. Dass diese Pathologien bei den Indigenen nicht auftraten, erklärte er sich damit, dass diese ausschließlich durch die Nase atmeten (Abb. 2). ${ }^{23}$ Eine kriti-

16 Diese Perspektive nahm John Ruskin Ende des Jahrhunderts ein, siehe Mark Frost, ,,The Circles of Vitality، Ruskin, Science, and Dynamic Materiality“, in: Victorian Literature and Culture 39, 2011, S. 367-383. Für diesen Hinweis danke ich Dr. Iris Wien.

17 Siehe Hartmut Böhme, „Das Volle und das Leere. Zur Geschichte des Vakuums“, in: Bernd Busch (Hg.), Luft (=Schriftenreihe Forum Bd. 12: Elemente des Naturhaushalts IV), Bonn 2003, S. 42-66.

18 Siehe exemplarisch zur Luftforschung Adey 2014 (wie Anm. 1) und Bernadette Bensaude-Vincent, „Lavoisier: Eine wissenschaftliche Revolution“, in: Michel Serres ( $\mathrm{Hg}$.), Elemente einer Geschichte der Wissenschaften, Frankfurt am Main 21995 [1994], S. 643-685.

$19 \mathrm{Zu}$ den pneumatischen Technologien wie Vakuumpumpen und anderen Apparaturen siehe Connor 2010 (wie Anm. 1), S. 14-101 und zu Wrights Gemälde Matthew Craske, „An Experiment on a Bird in the Air Pump“, in: Ausst.-Kat.
Bristol 2017 (wie Anm. 5), S. 22-32 sowie das Gespräch zwischen Anselmo Fox und Friedrich Weltzien in diesem Band.

$20 \mathrm{Zu}$ den Experimenten und einigen Abbildungen siehe Connor 2010 (wie Anm. 1), S. 69, S. 73, S. 111 und S. 163. 21 Ebd., S. $106 f$.

22 Christopher Grainge, „Breath of Life. The Evolution of Oxygen Therapy“, in: J R Soc Med 97, 2004, S. 489-493.

23 Für eine kritisch-historische Einordnung von Catlins künstlerischer, sammlerischer, anthropologischer und ethnographischer Praxis vor dem Hintergrund des imperialen Projekts der USA siehe Stephanie Pratt, „Integrating the ,Indian': the Indigenous American collections of George Catlin and Paul Kane“, in: Daniel J. Rycroft (Hg.), World Art and the Legacies of Colonial Violence, London 2016, S. 59-82 sowie George Catlin. American Indian Portraits, Ausst.-Kat. National Portrait Gallery London 2013. 


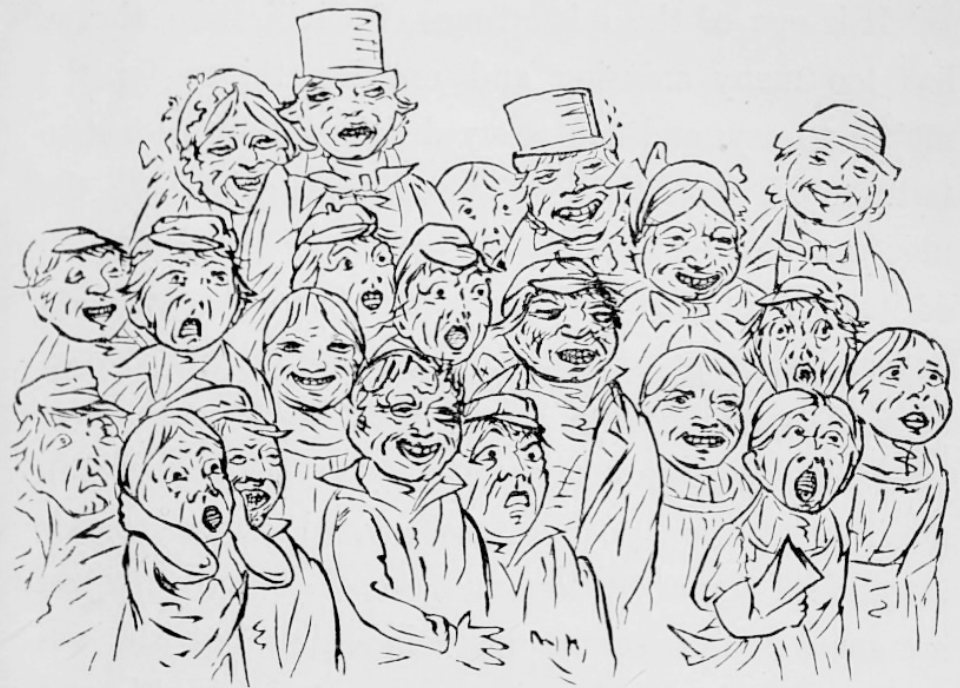

as familiar in our streets, or as it would be viewed by an equal multitude of savage childien.

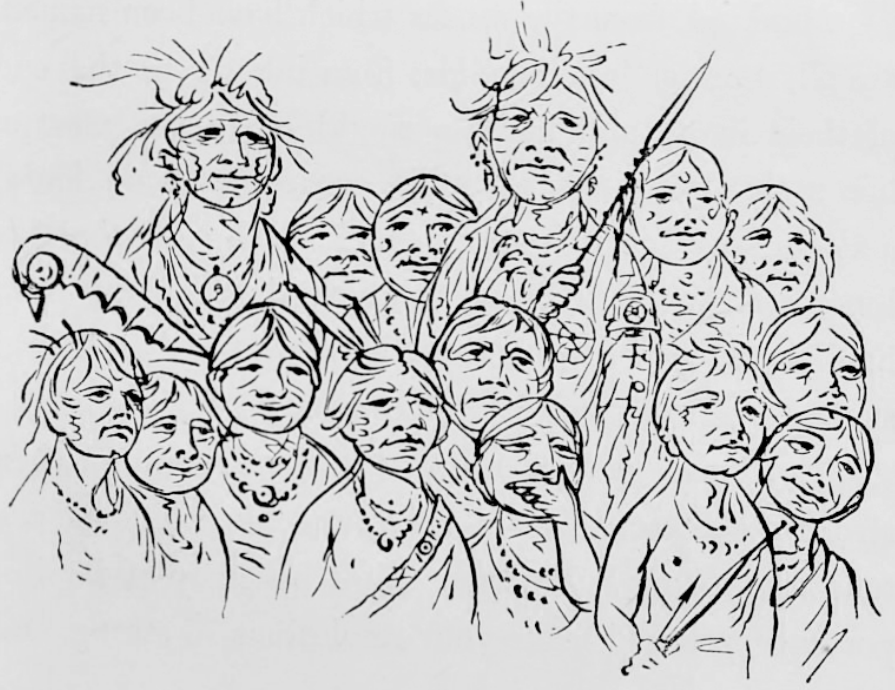


sche, postkoloniale Untersuchung von Catlins ethnographisch motivierten Porträts mit Fokus auf die spezifischen Darstellungen von geschlossenen Mündern, geweiteten Nasenflügeln und anderen Gesichtsmerkmalen steht noch aus. An ihnen ließe sich diskutieren, wie subtil und zugleich ideologisiert Atmung bildlich in Erscheinung treten kann.

In anderen Texten von Künstler*innen seit dem frühen 19. Jahrhundert wurde derweil dezidiert über die Atemluft in Gemälden nachgedacht, insbesondere in Deutschland: So beschrieb Johann Wolfgang von Goethe die Gemälde des französischen Malers Claude Lorrain aus dem 17. Jahrhunderts als „duftige“ Landschaften“. ${ }^{24}$ Die ,duftige Luft" ist dabei unter anderem als Synonym für die gereinigte Luft zu verstehen: ${ }^{25}$ Luft trete bei Claude als leicht bewegte, ,ventilierte' Atmosphäre auf, die als reine, nicht stagnierende, also sich erneuernde Luft konzipiert ist - als sonnendurchflutet, transparent und raumgreifend. ${ }^{26}$ Bislang weitestgehend unerforscht sind die mit solchen Atmosphäre-Konzeptionen verbundenen Vorstellungen der Zeitgenoss*innen, in Bildern zu atmen: „Wir glauben die balsamischen Düfte eines reizvolleren Klima's zu athmen“, schrieb etwa Carl Seidel um 1825 über das ideale Landschaftsgemälde. ${ }^{27}$ Den Atemprozess übertrug auch Carl Gustav Carus auf die Malerei, und dies sowohl produktions- als auch wirkungstheoretisch. Carus erörterte, wie sich vor Claudes und Jakob van Ruisdaels Landschaftsbildern die Atmung verändere und lobte die gleichsam reine Luft in deren Werken. ${ }^{28}$ Im Kontrast dazu beschrieb er weiterhin, wie er sich beim Malen von trüber Luft emotionaler Trübungen entledigen und so seine Lebensgeister wecken konnte: Der Malvorgang wird hier gleichsam zu einer reinigenden Ausatmung ästhetisiert. In diesem Sinne trat der Atem im 19. und 20. Jahrhundert häufig als ein Problem künstlerischer Lebensführung, als Inspirationsquelle, aber auch als Hindernis für die kreative Tätigkeit auf.

In Künstlerschriften des frühen 20. Jahrhunderts stellt die Ansteckung mit einer bestimmten Luft ein wiederkehrendes Thema dar. ${ }^{29}$ Das Eingeatmete wird dabei sowohl physikalisch wie auch im übertragenen

24 Gerhard Hard, ,„Dunstige Klarheit.' Zu Goethes Beschreibung der italienischen Landschaft, in: Die Erde. Zeitschrift der Gesellschaft für Erdkunde 100, 1969, S. 138-154; Joseph Vogl, „Luft um 1800“, in: Armen Avanessian/Winfried Menninghaus/Jan Völker (Hg.), Vita aesthetica: Szenarien ästhetischer Lebendigkeit, Zürich 2009, S. 45-53.

25 Alain Corbin, Pesthauch und Blütenduft. Eine Geschichte des Geruchs, Berlin 2005 [1984], S. 95-101.

26 Frances Gage, „Chasing ,Good Air‘ and Viewing Beautiful Perspectives: Painting and Health Preservation in Seventeenth-Century Rome“, in: Sandra Cavallo/Tessa Storey (Hg.), Conserving Health in Early Modern Culture. Bodies and Environments in Italy and England, Manchester 2017, S. 237-261, hier: S. 251-255.
27 Seidel zit. n. Annik Pietsch, Material, Technik, Ästhetik und Wissenschaft der Farbe 1750-1850. Eine produktionsästhetische Studie zur ,Blüte' und zum ,Verfall' der Malerei in Deutschland am Beispiel Berlin, Berlin u.a. 2014, S. 460.

28 Carl Gustav Carus, Neun Briefe über Landschaftsmalerei (1815-1824), Dresden 1995, S. 76 und S. 162.

29 Siehe zu diesem Prinzip und Ästhetiken der Ansteckung weiterführend Mirjam Schaub/Nicola Suthor/Erika Fischer-Lichte (Hg.), Ansteckung. Zur Körperlichkeit eines ästhetischen Prinzips, München 2005. Das Prinzip der Ansteckung, das aufgrund des Coronavirus 2020 so rasch wie nachhaltig ins Zentrum öffentlicher Aufmerksamkeit rückte, ist jedoch nicht rein naturwissenschaftlich und medizinisch zu denken. Darauf weisen Ästhetiken der 
Sinne als soziale Luft gedacht. Im Rekurs auf die antike Miasmen-Theorie, welche sich über das Mittelalter bis in die Moderne als populäre Vorstellung tradiert hat, ${ }^{30}$ thematisierten Künstler wie Marcel Duchamp und František Kupka in Briefen und anderen Texten sich wandelnde soziale und künstlerisch-kreative Atmosphären, die man einatmete und unter denen man entweder litt oder auflebte. ${ }^{31}$

Im Konzept der ,entarteten Kunst', das seine Wurzeln in der Romantik hat, werden Entartung und Degeneration ebenfalls wiederholt mit verschmutzter Luft in Verbindung gebracht. ${ }^{32}$ Der Literaturwissenschaftler Carl Weitbrecht bescheinigte um 1912 der jungen Generation deutscher Literaten, dass sie der "schlimmsten Krankenluft“ entstammten. ${ }^{33}$ Die Schriftsteller hätten den Fehler begangen, die „Türen und Fenster weit auf[zureißen], um die vermeintlich gesunde Luft des Auslandes hereinzulassen“, die jedoch „nur neue Krankheitskeime mitbrachte". ${ }^{34}$ Die Obsession für Gesundheit und Reinheit im physischen und gesellschaftlichen Sinne hat somit mindestens zwei Seiten: Sie umfasst „Technologien des Selbst“" ${ }^{35}$ zur Kultivierung des eigenen Lebens und der Kreativität, ebenso wie die systematische Degradierung anderer Kulturen und die Vernichtung von Leben, das als ,unwürdig‘ gekennzeichnet wurde.

Angesichts der Verschlechterung von Luftqualität und Lebensbedingungen aufgrund von Industrialisierung, Urbanisierung und Bevölkerungswachstum entwickelten sich in Europa und in den USA Lebensreform- und Naturheilbewegungen, in denen das freie Atmen sowie Atemtechniken unterschiedlicher, z.B. asiatischer Provenienzen eine wesentliche, wenngleich in der Geschichtsschreibung kaum beachtete Bedeutung einnahmen. Nicht nur durch die Gründung von Kommunen auf dem Lande, auch durch Reformkleidung, Rhythmus- und Tanzbewegung rückte der Atem als Chiffre für Befreiung und Gesundheit ins Zentrum von Kunst und Kultur. ${ }^{36}$ Soziale Architekturutopien wie die Gartenstadtbewegung sowie die Entwicklung neuer Technologien wie

Ansteckung hin, die vor allem in den Kulturwissenschaften und zunehmend in den 2000er Jahren als Metapher und positiv besetztes Prinzip erforscht wurden (ebd., S. 10f.). Parallel zu frühen Impfexperimenten bildeten sich im Frankreich des 18. Jahrhunderts Theorien heraus, welche die schmerzliche Affizierung durch die Kunst - die Hervorrufung von Symptomen ohne reale Krankheit - als Ideal bestimmten (ebd., S. 14).

30 Ebd., S. 16.

31 Vgl. den Beitrag von Lars Blunck in diesem Band sowie Linn Burchert, Das Bild als Lebensraum. Ökologische Wirkungskonzepte in der abstrakten Kunst, 1910-1960, Bielefeld 2019, S. 242-244.

32 Vgl. Adey 2014 (wie Anm. 1), S. 187.

33 Zit. n. Dina Kashapova, Kunst, Diskurs und Nationalsozialismus, Dissertation a. d. Universität Tübingen 2006, S.62.
34 Ebd.

35 Michel Foucault, „Technologien des Selbst“, in: ders., Ästhetik der Existenz. Schriften zur Lebenskunst, hrsg. v. Daniel Defert/Francois Ewald, Frankfurt am Main 2007 [1982], S. 287-318, hier: S. 289.

36 Vgl. exemplarisch die Verweise zu Arthur B. David und Fidus weiter unten. Siehe weiterführend Ute Ackermann, „,Bodies Drilled in Freedom': Nudity, Body Culture, and "Classical Gymnastics at the Early Bauhaus", in: Elizabeth Otto/Patrick Roessler (Hg.), Bauhaus Bodies: Gender, Sexuality, and Body Culture in Modernism's Legendary Art School, London u.a. 2019, S. 25-47; Gabriele Brandstetter, „Rhythmus als Lebensanschauung. Zum Bewegungsdiskurs um 1900“, in: Christa Brüstle et al. (Hg.), Aus dem Takt. Rhythmus in Kunst, Kultur und Natur, Bielefeld 2005, S. 33-44. 
das Air-Conditioning machen deutlich, wie zentral Luftqualität und Atmung in gesellschaftliche Entwicklungen, Design und Imagination integriert wurden. ${ }^{37}$

Zugleich ist die Moderne die Epoche pseudomedizinischer Rassentheorien und des terroristischen Einsatzes von Gasen: Ende April 1915 kam zum ersten Mal das tödliche Senfgas in den Schützengräben des Ersten Weltkrieges zum Einsatz, über zwanzig Jahre später begann der systematische Mord durch Gas in den Konzentrations- und Vernichtungslagern der deutschen Nationalsozialisten. ${ }^{38}$ In beiden Fällen handelt es sich um Ereignisse, die nicht über visuelle Dokumente der Zeit vermittelt, sondern nachträglich in Filmen, Gedenkstätten sowie durch Objekte wie Gasmasken erinnert werden. Peter Sloterdijk thematisiert ausgehend vom Giftgaskrieg eben jene „Kämpfe“ und „Traumata“, welche den „menschliche[n] Aufenthalt in atembaren Milieus zu einem Gegenstand expliziter Kultivierung“" werden ließ. ${ }^{39}$ Im Gaskrieg offenbaren sich gemäß Sloterdijk paradigmatisch drei zentrale moderne Zugriffe auf die Luft: die „Praxis des Terrorismus“, das „Konzept des Produktdesigns“" und neue „Umweltgedanken“. ${ }^{40}$

Die Zeit des Kalten Krieges schließlich war sowohl vom Design innovativer Atemtechnologien wie auch von neuen Zugängen zur Umwelt geprägt: Der Wettlauf zum Mond und die Reisen ins Vakuum brachten eine reiche, hochpolitisierte visuelle Kultur des Atems in Design, Film und Technologie hervor. ${ }^{41}$

Gegenwärtig wird der Atem vermehrt in das Zentrum interaktiver, transdisziplinärer Kunst- und Designpraktiken gestellt, die auf ästhetischen Überlegungen, wissenschaftlichen Experimenten und aktuellen Übertragungs- sowie Feedback-Technologien aus dem Gesundheitsund Lifestyle-Bereich basieren. ${ }^{42}$ Diese Kunst- und Designformen bauen zum einen auf die Performance-Kunst und Body Art seit den 1960ern auf. ${ }^{43}$ Zum anderen sind sie undenkbar ohne die Kybernetik, die sich seit den 1940er Jahren als Fachgebiet etabliert und spätestens mit dem

37 Colin Porteous, The New eco-Architecture: Alternatives from the Modern Movement, London 2002; siehe außerdem den Beitrag von Ole W. Fischer in diesem Band.

38 Siehe Beitrag von Kerstin Borchhardt in diesem Band.

39 Peter Sloterdijk, Schäume (= Plurale Sphärologie, Band 3), Frankfurt am Main 2004, S. 88, siehe außerdem S. 100103 zur Gasmaske als Designobjekt und den „Kampfgiftwolken als ,produktdesignerische[r] Aufgabe““. Zu Atemtechniken und Atemtechnologien der Moderne siehe weiterführend John Durham Peters, „The Media of Breathing“, in: Lenart Škof/Petri Berndtson (Hg.), Atmospheres of Breathing, New York 2018, S. 167-195.

40 Sloterdijk 2004 (wie Anm. 39), S. 89.

41 Siehe Beitrag von Marcus Becker in diesem Band.

42 Stefanie Heine, „Breathing Machines. Inspiration and Interdependence in Contemporary Art Installations“, in: Marc Carduff/Stefanie Heine/Michael Steiner ( $\mathrm{Hg}$.), Die Kunst der Rezeption, Bielefeld 2015, S. 69-85. Für eine umfangreiche, allerdings nicht wissenschaftlich-kritisch durchdrungene Übersicht siehe Christina Grammatikopoulou, Encounters on the Borders of the Immaterial. Body, Technology and Visual Culture. Art and Breath (1970-2012), Dissertation a. d. Universitat de Barcelona 2013, Englische Version verfügbar unter: http://diposit. ub.edu/dspace/bitstream/2445/46750/2/02.C_GRAMMA TIKOPOULOU_ENGLISH_TEXT.pdf (letzter Zugriff: 20.7.2020); Quinlivan 2012 (wie Anm. 6).

43 Vgl. Schönsee 2013 (wie Anm. 4), S. 263-296 und Linn Burchert, „Inspiration und Exspiration. Atemsteuerung in künstlerischen Praktiken seit 1900“, in: Ästhetische Fremdbestimmung (=Kritische Berichte 3/2019), hrsg. v. Katja Müller-Helle/Julian Blunk, S. 44-55. 


\section{Linn Burchert}

Atem. Künste, Technologien und Architekturen der Moderne und Gegenwart
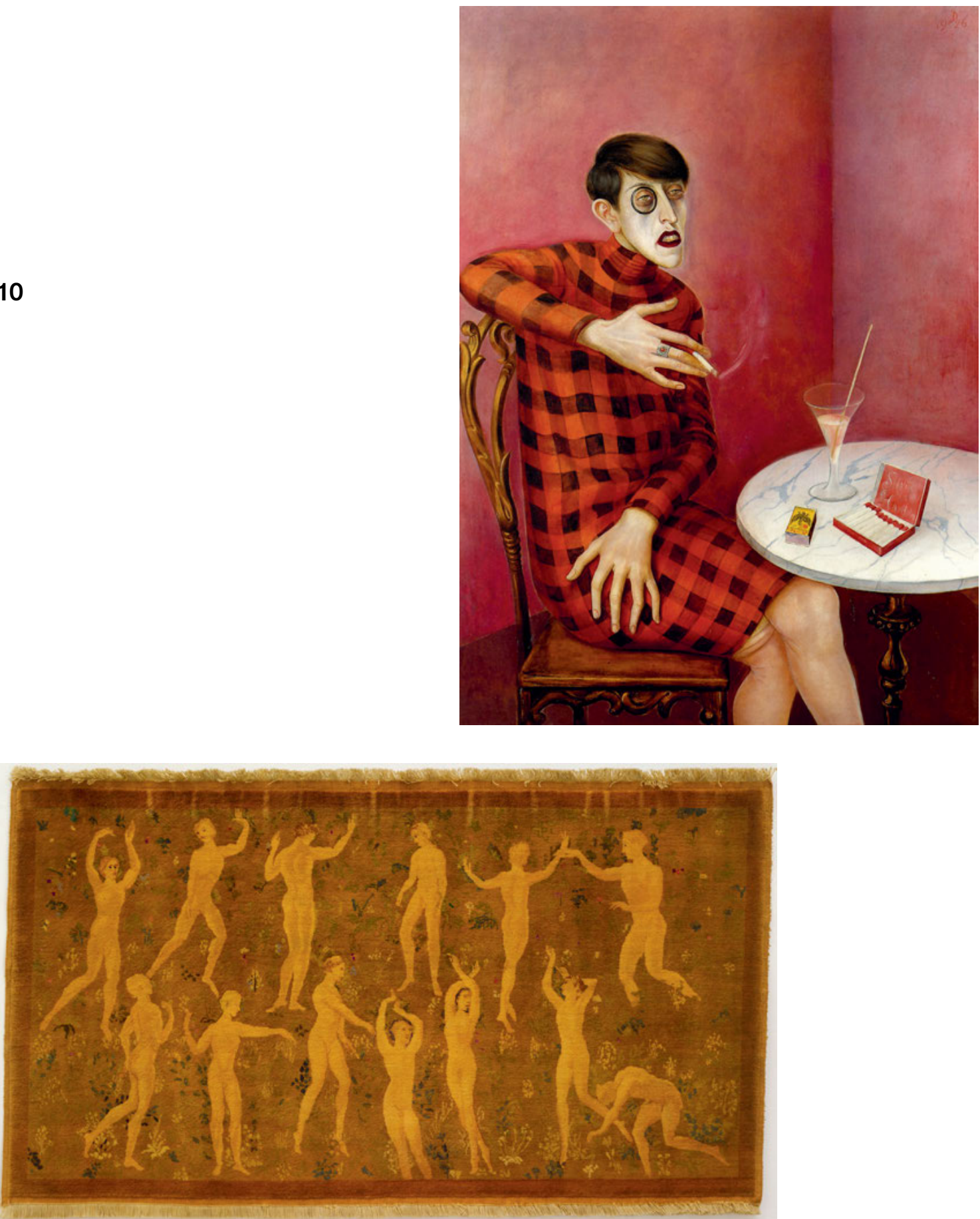

Abb. 3: Otto Dix, Porträt der Journalistin Sylvia von Harden, Mischtechnik auf Holz, $121 \times 89$ cm, 1926, Paris, Musée d'Art Moderne, Centre Georges Pompidou 


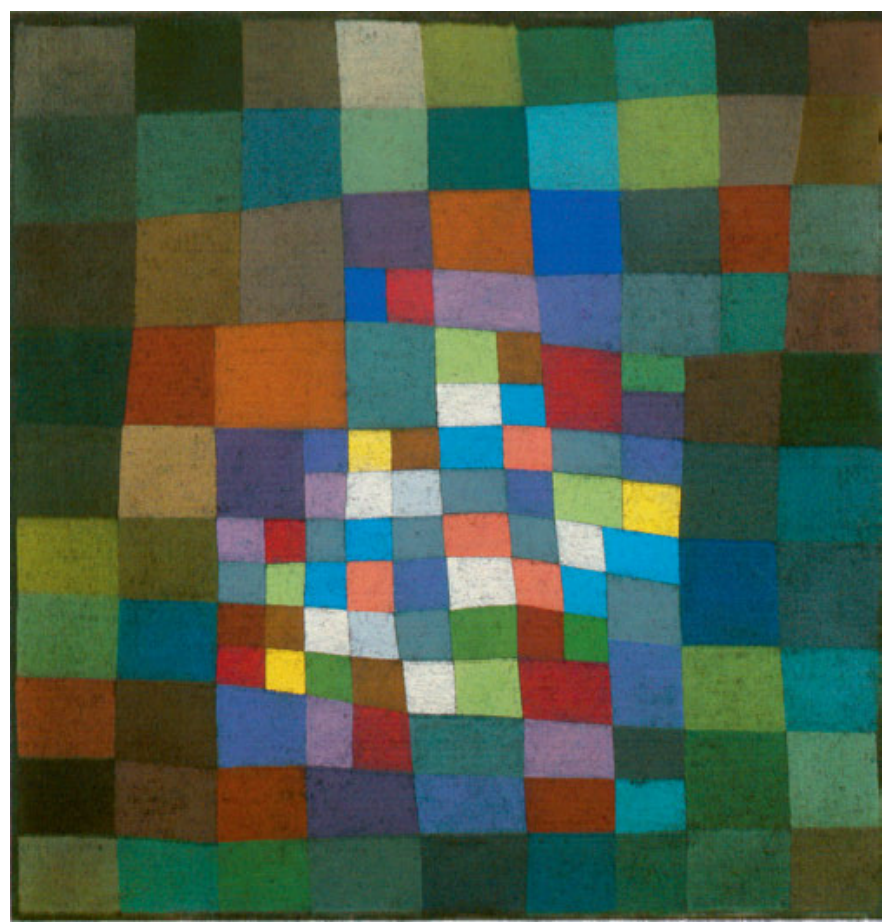

Abb. 5: Paul Klee, Blühendes, 1934, Öl auf Leinwand, $82 \times 80 \mathrm{~cm}$, Kunstmuseum Winterthur 
ausgehenden 20. Jahrhundert die Lebensrealitäten der Menschen radikal und zunehmend global verwandelt hat. ${ }^{44}$

Die Beiträge des vorliegenden Bandes beziehen sich vielfältig auf die dargelegten Aspekte: Sie untersuchen den Atem als Visualisierungsproblem, wissenschafts- und diskurshistorisch, praxisgeschichtlich mit Blick auf kreativ-künstlerische Produktionsprozesse, Techniken und Technologien sowie als Ausgangspunkt für Designideen und architektonische Experimente. Im Folgenden wird eine Übersicht über die einzelnen Sektionen und Beiträge gegeben und eine Verortung im breiteren Forschungskontext vorgenommen..$^{45}$

\section{Visuelle Evokationen des Atems}

Die Atmung drängt sich visuell nicht auf. Dennoch hat der Atem als Bildmotiv eine lange Geschichte: Er zeigt sich als göttlicher Hauch oder Strahl in mittelalterlichen und neuzeitlichen Tafeln ${ }^{46}$ in Seifenblasen als Vanitas-Motiv und als Sinnbild des sterblichen Menschen und somit des Lebens überhaupt. ${ }^{47}$ Ebenso präsentiert er sich in der künstlerischen Auseinandersetzung mit optischen Experimenten und Erkenntnissen des 17. und 18. Jahrhunderts. ${ }^{48}$ Die von einem Hohlkörper ausoder in ihm eingeschlossene Luft stellt so ein tradiertes und populäres Motiv dar, das in Moderne und Gegenwart neue Bedeutungen angenommen hat. Dass Atemdarstellungen beispielsweise keineswegs genderneutral sind, zeigte andernorts Barbara Langes Deutung von Otto Dix' Porträt der Journalistin Sylvia von Harden (1926). Die dargestellte Journalistin - androgyn und kantig, lässig die Zigarette in ihrer rechten Hand haltend, den Mund leicht im Ausatmen geöffnet - versteht Lange als Eroberin „des männlich konnotierten Geruchsterrains Tabak für sich" (Abb. 3). ${ }^{49}$

Während das Rauchen in Kunst und visuellen Medien ein tradiertes und hochgradig ästhetisiertes Motiv darstellt, ${ }^{50}$ hat die Kunstge-

44 Thomas Rid, Maschinendämmerung. Eine kurze Geschichte der Kybernetik, Berlin 2016, S. 10-16; siehe auch den Beitrag von Gloria Sutton in diesem Band.

45 Die Namen der Beitragenden sind in Abgrenzung zu den zusätzlichen Nennungen durch Kursivierung hervorgehoben.

46 Heine 2015 (wie Anm. 42), S. $75 f$. und Baert 2016 (wie Anm. 6).

47 Siehe Anita Hosseini, Die Experimentalkultur in einer Seifenblase. Das epistemische Potenzial in Chardins Malerei, Paderborn 2017, S. 34-44 und Karin Leonhard/ Sandra Hindriks, „Windhauch, [...] Windhauch. Das ist alles Windhauch. Zu einer Neubewertung des VanitasStilllebens", in: 21: Inquiries into Art, History, and the Visual 1 (1), 2020, S. 127-180.

48 Hosseini 2017 (wie Anm. 47), S. 59-63; vgl. auch das Gespräch von Anselmo Fox und Friedrich Weltzien in diesem Band.
49 Barbara Lange, „Duften, Stinken, Beissen. Geruchssinn und bildende Kunst“, in: Andrea Gottdang/Regina Wohlfarth (Hg.), Mit allen Sinnen: Sehen, Hören, Schmecken, Riechen und Fühlen in der Kunst, Leipzig 2010, S. 105119, hier: S. 116. Siehe weiterführend zur Ikonografie des Duftes seit dem Mittelalter im Kontext theologischer sowie sozial- und kulturhistorischer Entwicklungen: dies., „Sinnenwandel. Ein kleiner Streifzug durch die Geschichte und Duft und Geruch in der Kunst", in: Ausst.-Kat. Basel 2015 (wie Anm. 10), S. 97-104.

50 Siehe Thank you for smoking. Die Zigarette im Film, Kat., hrsg. v. Deutschen Filminstitut Frankfurt am Main, München 2014 zu den vielfältigen, ebenfalls genderspezifischen Dimensionen des Rauchens im Film. Siehe außerdem die Überlegungen von Anselmo Fox und Friedrich Weltzien in diesem Band zum Michelin-Kompressor. 
schichte einer anderen Form des exzentrischen Ein- und Ausatmens bislang weniger Beachtung geschenkt: dem Gähnen. Der Beitrag von Lisa Hecht in diesem Band deutet anhand dieses Reflexes auf die vielfältigen kulturhistorischen und gendertheoretischen Dimensionen des Atems hin. Edgar Degas' wiederkehrende Darstellungen von gähnenden Büglerinnen in den 1880er Jahren ordnet Hecht in eine entstehende Bildkultur der Hysterie ein, in welcher die Pathologisierung der weiblichen Langeweile im ausgehenden 19. Jahrhundert augenscheinlich wird. Vor dem Hintergrund des weit geöffneten Mundes der Büglerinnen perspektiviert die Autorin Fragen des Dekorumsverstoßes und der Bildwürdigkeit neu.

Nicht nur die Bildwürdigkeit sowie künstlerische, geschlechtliche und gesellschaftliche Grenzüberschreitungen, auch das Inkarnat als zentrale Darstellungsaufgabe der Malerei lässt sich unter dem Aspekt der Atmung neu beleuchten. So untersuchen Isabelle Schwarz und Pamela Bannehr in natur-, kunst- und kulturwissenschaftlicher Perspektive den Farbauftrag in Gemälden der Neuen Sachlichkeit unter dem Aspekt einer unterbrochenen Kommunikation mit der Außenwelt. Im atmosphärelosen Bildraum von Gerta Overbecks Bildnis Toni Overbeck scheint die Hautoberfläche versiegelt. Tonis Haut wird zur reinen Oberfläche maskiert, wodurch jegliche Entäußerung und Kommunikation unterbrochen scheint und eine Ästhetik entsteht, die dezidiert nicht darauf angelegt ist, eine Verbindung zu den Betrachtenden herzustellen. So eruieren die Autorinnen, was es bedeutet, wenn Atmung konzeptuell aus dem Porträt ausgeschlossen ist.

Die häufig zu beobachtende Starre und Leblosigkeit in den Bildwelten der Neuen Sachlichkeit stellt eine deutliche Gegenbewegung zur emphatischen Lebensbejahung der Lebensreformbewegungen um und nach 1900 dar. Der US-amerikanische Künstler Arthur B. Davies etwa rückte Atem und Vitalität ins Zentrum seiner Figurendarstellungen. Vergleichbar mit Lichtgebet (1910) des deutschnationalen Künstlers Fidus ${ }^{51}$ repräsentierte Davies atmende Körper in verschiedenen Stadien des Streckens und Weitens (Abb. 4). ${ }^{52}$ Den sogenannten „lift of inhalation" als ästhetische Kategorie theoretisierte Davies anhand figurativer Darstellungen ,einatmender Körper seit der Antike. ${ }^{53}$

51 Jost Hermand, Avantgarde und Regression. 200 Jahre deutsche Kunst, Leipzig 1995, S. 72-89.

52 Robin Veder, „The Joy of Breathing: Physical, Emotional, and Spiritual Uplift in the Art of Arthur B. Davies“, in: Mary D. Edwards/Elizabeth Bailey (Hg.), Gravity in Art, Jefferson u.a. 2012, S. 198-211.
53 Es handelt sich v.a. um Figuren, deren Zehen, Brustkorb, Arme und Kopf sich heben bzw. weiten. Siehe ebd., S. 198f. Zu zentralen Werken gehören weiterhin Salute to the Sun (1919), Hickory Grove (1924), Supplication (1919-20), Morning Quiet (1919-20). 
In einem ähnlich lebensbejahenden Geist und zeitlich parallel begannen Künstler*innen im frühen 20. Jahrhundert mit dem Überschreiten der Grenzen des Darstellbaren. Durch Abstraktion und Ungegenständlichkeit sollten unmittelbare, synästhetische Wirkungen erzeugt und Einschränkungen des Bildmediums überwunden werden. So problematisiert auch Lange andernorts, dass Geruch als wesentliches lebensweltliches Phänomen nur vermittelt - etwa durch eine besondere "Zeichensprache der Malerei“ thematisch werden könne ${ }^{54}$ - man denke an das Motiv der Blume im höfischen Porträt des 18. Jahrhunderts als Chiffre für den Wohlgeruch. ${ }^{55}$ Darüber hinaus weist Lange auf Formen der Abstraktion in Bildern hin, die ohne konkreten Gegenstandsbezug als Geruch konnotiert sein können. So seien in der Kunstkritik des 19. Jahrhunderts ein „heftiger Duktus und reliefartige Oberflächenstrukturen mit den als undisziplinierbar verstandenen Sinnesreizen Geschmack und Geruch in Verbindung gebracht [...] und als Sinnenrausch verstanden" worden. ${ }^{56}$

Anfang des 20. Jahrhunderts schließlich phantasierte der Futurist Umberto Boccioni, dass die „Werke der Maler“ bald „wirbelnde Architekturen von Tönen und Gerüchen aus farbigen Gasen sein“ würden. ${ }^{57}$ Auch Wassily Kandinsky, Paul Klee, Johannes Itten, Mark Rothko und Andere begriffen ihre Bilder als Atem- und Lufträume, denen mitunter synästhetisch-olfaktorische Wirkungen zugesprochen wurden. ${ }^{58} \mathrm{Sie}$ sollten beispielsweise durch eine rhythmische Gestaltung selbst zum Atmen gebracht werden und die Betrachtenden in ihrem Rhythmus atmen lassen. ${ }^{59}$ Atem wird hier abstrahiert zur Wirkung von Farbflächen und fungiert als Mittel der Kommunikation der Betrachtenden mit dem Werk (Abb. 5).

Es liegt nahe, diese Bildkonzepte als Vorläufer späterer immersiver Installationskunst mit den Medien Licht, Luft und Ton zu betrachten, welche die zeitgenössische Kunst maßgeblich prägen. Allerdings ist die visuelle Geschichte des Atems keineswegs als eine Fortschrittsgeschichte von der Figuration zur Abstraktion, hin zu Ungegenständlichkeit und schließlich zum Erschaffen von realen Räumen zu fassen. Dem Problem der Darstellung des Atems widmen sich zeitgenössische Künstler*innen ebenfalls, indem sie beispielsweise Formen der Figuration, der Abstraktion und der Textualität im Medium des Comics miteinan-

54 Lange 2010 (wie Anm. 49), S. 116. Mit Blick auf die Vormoderne geht Lange auf Giottos Auferweckung des Lazarus (1313) sowie Giorgio Vasaris Darlegungen zu Leonardos Prunkschild mit dem Kopf der Medusa ein, deren stinkender Atem hier illusionistisch vergegenwärtigt werde (ebd., S. 98-100).

55 Ebd., S. $103 f$.

56 Ebd., S. 116.
57 Umberto Boccioni, Futuristische Malerei und Plastik (Bildnerischer Dynamismus) [1913], hrsg. und übers. v. Astrid Schmidt-Burkhardt, Dresden 2002, S. 199; vgl. Burchert 2019a (wie Anm. 31), S. 218.

58 Vgl. Burchert 2019a (wie Anm. 31), S. 224f. und Lange 2010 (wie Anm. 49), S. 116f.

59 Vgl. exemplarisch Burchert 2019a (wie Anm. 31), S. 261-273, S. 305-312 und S. 317-323. 
der verbinden. Maria Weilandts Stichprobe in unterschiedliche Ausformungen des zeitgenössischen Comics zeigt die Vielfalt auf, wie Atmung innerhalb von Figurationen sichtbar gemacht und maßgeblich in die Konstruktion von Bedeutungen einbezogen wird. Jedes von Weilandts Fallbeispielen verweist dabei auf die eigentliche „Unsichtbarkeit und damit Undarstellbarkeit von Atemluft“, wenn Wölkchen, sprachliche Bilder und geometrische Formen in Erscheinung treten, um die spezifische physikalische Gegenwart des Atems greifbar zu machen.

In ihrer transdisziplinären, experimentellen Auseinandersetzung mit der historischen Schlieren-Apparatur widmen sich Iva Rešetar und Clemens Winkler der Sichtbarmachung von Strukturen und Bewegungen fluider Körper in der vermeintlichen Leere. Ihre Bildreihe visualisiert Unterschiede in Dichte, Temperatur und Druck, die in der Luft auftreten, wenn beispielsweise Gesten des Atmens, Sprechens sowie Prozesse des Ausströmens und der Verdunstung vor dem SchlierenSpiegel stattfinden. Im Gespräch mit Léa Perraudin reflektieren sie ihre Experimente und deren Implikationen: Was zuvor u. a. als Erkenntnisinstrument in den Naturwissenschaften diente, wird hier zu einer poetischen und affektiven Praxis umgedeutet, welche gängige Konzeptionen materieller Grenzen hinterfragt. So zeigt sich hier die Leere als ein offener Ort, erfüllt von Austauschprozessen und Überschreitungen. Die Photographien scheinen eine Intensivierung des Wahrnehmbaren zu ermöglichen und machen zugleich die Grenzen der menschlichen Wahrnehmung evident.

\section{Formgebende Atmung}

Der Atem ist somit nicht nur eine Herausforderung der Darstellung und Sichtbarmachung, er ist auch Teil künstlerischer, gestalterischer und experimenteller Produktionsformen. Sprichwörtlich ist der künstlerische Atem im Pygmalion-Mythos geworden, demzufolge der Künstler gottgleich seiner Skulptur Leben einhaucht. ${ }^{60}$ Der Habitus des Künstlers als Erzeuger von Leben wurde in der Moderne immer wieder aufgegriffen - zumeist seitens männlicher Künstler, welche die ausgesprochene Vitalität ihrer Werke proklamierten. ${ }^{61}$ Piero Manzoni und Marcel Duchamp hingegen haben eben diese Künstlermythen ironi- 
siert. In ihren Atemwerken offenbaren sich Form und proklamierter Inhalt als äußerst prekär.

Duchamps Glasampulle Air de Paris (1919) war, so deutet es andernorts Lars Blunck, Sinnbild einer altbekannten, ,uninspirierten Luft, die mit einem stagnierenden Künstlertum in Verbindung stand. ${ }^{62}$ Duchamps Air de Paris ebenso wie sein A quart of smoke exhaled from $M D$ 's mouth and of a havana cigar on June 15, 1965 at 10.45 a.m. in my apartment 7, via Baglivi, Rom, Italy/ Gianfranco Baruchello persiflierten den Künstleratem als vermeintlich göttliche Kraft. ${ }^{63}$ Die Rauchskulptur verflüchtigte sich äußerst schnell, zurück blieb eine Hülle, die heute keine Partikel der Duchamp'schen Atemluft mehr tragen dürfte. Über die Erweiterung bzw. Ironisierung des Kunstbegriffs hinaus wird hier vor allem eines evident: der rasche physische Verfall von Luftkörpern. ${ }^{64}$ Den Museumsstücken ist längst die Luft ausgegangen, der Atem ließ sich darin nicht verewigen. Atem wird so evident als etwas Ephemeres, das im ständigen Austausch von Innen und Außen, Individuum und Umwelt begriffen ist, sich kaum einfangen lässt und stetig entweicht. Wie also kann er überhaupt zu Form- und Gestaltungsprozessen beitragen? Dieser Frage wird in den Beiträgen dieser Sektion anhand von kulturellen und künstlerischen Techniken der Formgebung nachgegangen.

Mit Patricia Ribaults Essay zu Traditionen der Glasbläserei und Weberei wird ein produktionstheoretischer Fokus auf das Problem der Formgebung durch die Atmung unternommen. Darüber hinaus reflektiert die Autorin Formen der Weitergabe kultureller Techniken und deren Bindung an einen bestimmten künstlerischen Habitus. Am Beispiel der Tradition sardinischer Weberei referiert Ribault auf die Atmung als magischer Akt zur Fertigstellung und Animation des Webstücks, das demnach erst durch die ritualartigen, ,magischen“ Handlungen der Weberin ihren Glanz erhält. Im Unterschied zum Pygmalion-Motiv untersucht die Autorin damit eine vornehmlich weiblich konnotierte Technik - eine Jahrhunderte alte Praxis, die sich nicht dem Impetus des männlichen Genies bedient, somit eine alternative Tradition künstlerischer Schöpfungsmythen anbietet und - wenig erstaunlich von der Kunst- und Bildgeschichte lange ignoriert wurde.

62 Lars Blunck, Duchamps Readymade, München 2017, S. 192.

63 Duchamp etwa hatte das Rauchen bzw. das Atmen überhaupt zur Kunst und zur idealen Tätigkeit eines ,ruheständlerischen‘ Künstlers erklärt. Dazu siehe ebd., S. 244-247. Zum Atmen als Lebenshaltung und Künstlerhabitus siehe den Beitrag von Blunck in diesem Band.
64 Gregory Tentler, „Best Most Sensational Balloons: Piero Manzoni's Corpo d'aria/Fiato d'artista“, in: California Italian Studies 6 (2), 2016, S. 1-27, insbes. S. 23-25. Die Überreste des Werkes können auf der Webseite von Tate London erahnt werden: https://www.tate.org.uk/ art/artworks/manzoni-artists-breath-t07589 (letzter Zugriff: 9.9.2020). 
Konkrete Techniken der Formgebung in der Webkunst untersucht der Beitrag von Mona Schieren anhand der textilen Werke der USamerikanischen Künstlerin Leonore Tawney. Die Autorin beleuchtet die Bedeutung der Körperarbeit und Atemtechnik des Sensory Awareness-Programms der Pädagogin Charlotte Selvers für Tawneys Werk motivisch, mit Blick auf den künstlerischen Produktionsprozess und als Erkenntnisform und -methode. Neben der Rezeption, amerindischer Kulturen identifiziert Schieren die Atemarbeit als zentralen Faktor für künstlerische Entwicklungen Tawneys. Damit schreibt sich die Künstlerin in eine reiche, aber wenig erforschte Tradition des 20. Jahrhunderts ein, Atem- und andere Körpertechniken als Vorbereitung oder Praxis der künstlerischen Tätigkeit einzubeziehen. Gertrud Grunow und Johannes Itten am Weimarer Bauhaus hatten etwa Atemübungen und Gymnastik als Voraussetzungen für die künstlerische Tätigkeit etabliert; ${ }^{65}$ und auch für Arthur B. Davies waren derartige Gesundheitspraktiken zentral.

Im Gespräch zwischen Friedrich Weltzien und dem Künstler Anselmo Fox wird der Atem als formgebende Kraft diskutiert, welche vielfältige visuelle Spuren und Gestalten erzeugen kann. Fox arbeitet mit aufblasbaren Materialien wie Ballons und Kaugummi. Sein Fokus auf verschiedene Medien und Techniken im Zweidimensionalen ebenso wie im Dreidimensionalen, lässt Gesetzmäßigkeiten und Eigenheiten der Atemluft, ebenso wie des Vakuums aufscheinen. Das Gespräch zeigt die enge Verbindung der Kulturgeschichte des Atems mit dezidiert männlichen und industriell-kapitalistischen Diskursen der Selbstbehauptung, Beherrschung und Selbstüberschätzung, die als Aktualisierung des heute antiquiert anmutenden Pygmalion-Mythos betrachtet werden können - Traditionen, zu denen die Beiträge von Ribault und Schieren Kritik und Gegenentwürfe formulieren.

\section{Atem, Identität, Affekt}

Ausgehend von den obigen Überlegungen zum künstlerischen Schaffensprozess mit dem Atem wird in der anschließenden Sektion dem Atem als Mittel zum Ausdruck von Identität zwischen den Polen der Souveränität und Abhängigkeit nachgegangen, wobei Prozesse der Abgrenzung einerseits und Gefühle der Empathie andererseits Bedeu-

65 Zur Bedeutung des Atmens und der Gesundheit für die

Produktion von Kunst vgl. Linn Burchert, „The Spiritual

Enhancement of the Body: Johannes Itten, Gertrud

Grunow, and Mazdaznan at the Early Bauhaus", in:

Elizabeth/Roessler 2019 (wie Anm. 36), S. 51-74. 
tung erlangen. Neben der produktionsästhetischen Perspektive wird die Atmung in der Wahrnehmung von Kunst in den Blick genommen. Dabei sind es insbesondere Affekte - unkontrollierbare Reaktionen auf Erlebnisse -, welche die vermeintliche Souveränität des Subjekts untergraben und so auch neue Reflexionen zu Identität anregen können.

In dem bereits erwähnten Artikel über das Gähnen bei Degas weist Hecht auf das Selbstporträt Joseph Ducreux' als Gähnender (1873) hin. Dieses Gemälde visualisiert den Gestus eines gekränkten Künstlers, dessen Gemälde für die Ausstellung des jährlichen Salon du Louvre abgelehnt wurde. ${ }^{66}$ Für Duchamp kann Lars Blunck eine vergleichbare Geschichte aufdecken: So habe der 1912 aus dem Salon des Indépendants ausgeschlossene Künstler 1921 mit seinem Flakon Belle Haleine (Schöner Atem) eine „,ironische Replik auf die Theoriebildungen der peinture pure" geschaffen, um seine Abgrenzung von dieser Gruppe zu markieren. Auf die Kränkung des Subjekts folgen die Rache und die unabhängige Selbstpositionierung als Künstler, die allerdings mit dem Modell künstlerischer Selbstherrlichkeit keineswegs bricht.

Die Atmung als Zeichen höchster individueller Souveränität untersucht Blunck am Beispiel von Duchamps proklamierter Lebensweise, die einerseits mit vermeintlicher Faulheit und Untätigkeit verknüpft ist, andererseits aber schöpferisches Potential birgt. Als spezifisches Verständnis eines kapitalistischen Liberalismus identifiziert Blunck Duchamps Haltung dem Leben gegenüber, welche den souveränen Atem, die Ungebundenheit und die absolute Individualität in ihr Zentrum stellt.

Anhand von Duchamp werden außerdem Bezüge zwischen Atmung und Biopolitik deutlich. Die Aushandlung der Grenzen individueller und staatlicher Kontrolle werden in Marta Smolińskas Aufsatz zur polnischen Gruppe Werkstatt der Filmform der 1970er Jahre konkret: Die Künstler Józef Robakowski und Paweł Kwiek rekurrierten in ihren Videoarbeiten auf den Atem als Marker von Sterblichkeit und Verletzlichkeit einerseits und als Mittel der staatlich-medialen Manipulation andererseits. Die Künstler setzten ihren Atem als indirekte Kritik an der sozialistischen Diktatur ein, indem sie beispielsweise auf eigenwillige Weise Formen der Medienmanipulation zitierten. Bei beiden Künstlern changiert der Atem dabei zwischen Zwangs- und Befreiungsgestus. 
Diese Dialektik von Freiheit und Zwang in der Atmung hat andernorts die Literaturwissenschaftlerin Lisa Siraganian erforscht: „Modernism [...] evoked breath and air to talk precisely about autonomy and invoke a person's varied freedoms, not to deny the body and its sensory experiences “ ${ }^{67}$ Siraganian geht es jedoch nicht um die Autonomie des Künstlersubjekts, wie sie etwa Duchamp für sich reklamierte, sondern um die Autonomie bzw. die Agency des Werkes, welche Autonomie in der Rezeption entweder erlaube oder nicht. Gertrude Steins Lyrik ohne Punkt und Komma mache beispielsweise keine Vorgaben, wann die Leser*innen in der Lektüre innehalten, ein- und ausatmen; Siraganian stellt sie somit in den Kontext einer spezifisch modernen liberalen Politik der vermeintlichen Wahlfreiheit. ${ }^{68}$ Dagegen setzt sie das Konzept der Verkörperung, welches Charles Olson seit den 1960er Jahren unter anderem zur Repräsentation migrantischer und afroamerikanischer Perspektiven in den USA zum Einsatz brachte, indem er seine poetischen Texte als spezifische Sprach- und Atemrhythmen gestaltete. ${ }^{69}$ Er wendet sich damit - allerdings als Weißer - gegen die Idee eines universalen, US-amerikanischen Subjekts, wenn er Konstruktionen individueller, minorisierter Perspektiven erfahrbar macht und diese durch seine Leser*innen verkörpern lässt.

Minorisierte Identitäten und Fragen der Verkörperung untersucht auch Barbara Oettl in ihrem Beitrag anhand von Werken Chris Burdens, Theresa Margolles' und Gregor Schneiders. In der Simulation existentieller Gefährdungen oder Tabu-brechender Thematisierungen des Todes liegt Oettl zufolge eine künstlerische Strategie, welche darauf abhebt, mittels Kunst die eigene Sterblichkeit unmittelbar erfahrbar zu machen. Oettl reflektiert unter Bezugnahme auf naturwissenschaftliche Erkenntnisse die Wirkungen der Kunst auf den Atem und somit die synästhetische, auf die Reflexe wirkende Kunsterfahrung als potentiell läuterndes Instrument der Selbstreflexion.

Die Verknüpfung der ästhetischen Kategorie der Katharsis mit dem Atem existiert nicht erst in der Gegenwartskunst. Mark Rothko formulierte die Idee, den Betrachter*innen seiner Werke den Atem zu rauben, sie mit Bildern zu konfrontieren, die eine Enge erzeugen, welche sobald sie den Bannkreis des Bildes verlassen - ein befreites und bewusstes Atmen erlaube. ${ }^{70}$ Die zeitgenössische Kunst schreibt solche

67 Siraganian 2012 (wie Anm. 4), S. 14.

68 Ebd., S. 7f. und S. 24-50.

69 Ebd., S. 139-168.

70 Burchert 2019a (wie Anm. 31), S. 261-273. 
Strategien in steigender Intensität fort. Oettls Auseinandersetzung mit dem Fluchtimpuls und dem Ekel ausgehend von vermeintlich kontaminierter Kunst eröffnet beispielsweise höchst provokative Elemente, wenn die Besucher*innen in Margolles' Installation In the Air (2003) geruchslosen Seifenblasen ausweichen, die aus dem Waschwasser von Opfern des anhaltenden mexikanischen Drogenkrieges stammen. Hier findet keine Solidarisierung statt, sondern eine Abgrenzung. In solchen Werken ist es stets das privilegierte Subjekt, das aus dem Alltag völlig unbekannte Erfahrungen in Form simulierter Gefährdungssituationen durchläuft. Diese Arbeiten sind zugleich in starkem Gegensatz zu anderen, harmonistischen Ästhetiken der Moderne und Gegenwart zu sehen, die sich beispielsweise an Atem- und Gesundheitstechniken orientieren. $^{71}$

In der Gegenwart hat sich eine neue Gattung von Partizipationskunst herausgebildet, die Design und Technologie in Form von Wearables zum Zwecke einer vermeintlich optimierten Selbsterfahrung von Subjekten vereint. ${ }^{72}$ Die Praktiken rekurrieren häufig auf Techniken der Meditation, aber auch auf kontrollierte Körpertechniken auf Grundlage der quantifizierenden Messung von Körperfunktionen wie Atmung und Puls. ${ }^{73}$ Stephanie Heine hinterfragt andernorts die diesen Werken oft inhärente Rhetorik der Befreiung und der emanzipierten Teilnahmeposition, indem sie das technologische Setup und die Interdependenz von Mensch und Maschine betont. ${ }^{74}$ Eben diese Interdependenz ist ein häufig anzutreffendes Thema der Gegenwartskunst: Christa Sommerer und Lauren Mignonneaus Mobile Feelings (seit 2001) etwa hebt auf den mediatisierten und dennoch - durch haptische und taktile Impulse auf Grundlage der Messung von Puls und Atmung - als unmittelbar wahrgenommenen Kontakt ab, der auch therapeutische Wirkungen entfalten soll. ${ }^{75}$ Es geht um eine intime Kommunikation, allerdings ganz über Technologien und ohne Hautkontakt.

Kommunikation ohne Hautkontakt ist auch Gegenstand des diese Sektion abschließenden Gesprächs zwischen Dorothée King und dem Künstler Matt Morris. Dessen Euvre entzieht sich der soeben skizzierten Extreme von Ästhetiken existentieller Bedrohung und ästhetischer Heilsversprechen. Doch auch er stellt mit seinen Atem-Werken

71 So etwa bei Davies, der ausgehend von modernen Tanzund Rhythmuspraktiken eine neue, befreite Körperlichkeit und das psychische Wohlbefinden durch den Atem in das Zentrum seiner Kunst stellte. Siehe Veder 2012 (wie Anm. 52), S. 207.

72 Siehe weiterführend Felix Maschewski/Anna-Verena Nosthoff, Die Gesellschaft der Wearables: Digitale Verführung und soziale Kontrolle, Berlin 2019.

73 Dazu vgl. Grammatikopoulou 2013 (wie Anm. 42).
74 Heine 2015 (wie Anm. 42), S. 76f. Eben dieser kritische Zugang fehlt bei Grammatikopoulou 2013 (wie Anm. 42), die zweifelsohne die größte Sammlung atembezogener Arbeiten seit den 1970er Jahren zusammengetragen hat, jedoch v.a. deskriptiv mit den Werken umgeht und dabei die Werkdeutungen der Künstler*innen übernimmt.

75 Christa Sommerer/Laurent Mignonneau, „Mobile Feelings“, in: Bakke 2006 (wie Anm. 2), S. 38-43. 
Fragen von Identität, Alterität und Affekt. Morris setzt auf eine subtile Verwirrung durch seine Parfumkunst, die von der erotischen Schönheit des Duftes und deren Potential zeugt, sowohl angenehme Erinnerungen und Emotionen zu wecken, als auch xenophobe Affekte hervorzurufen. Im Gespräch zwischen King und Morris werden so zum einen die höchst individuellen Wahrnehmungen in der Erfahrung von Düften, zum anderen deren manipulative sowie unmittelbar affektive Wirkungen betont.

\section{Unterdrückte Atmung und toxische Atmosphären}

Seit dem 18. Jahrhundert sind Technologien der Manipulation, Messung und Lagerung von Luft omnipräsent. Künstlerische Arbeiten mit Duft wären ohne diese vielfältige Technologie- und Wissenschaftsgeschichte undenkbar. ${ }^{76}$ Die Aufsätze der vierten Sektion erweitern wesentliche Aspekte der vorangegangenen Beiträge auf künstlerische und technologische Auseinandersetzungen mit dezidiert lebensfeindlicher - kontaminierter oder schlichtweg fehlender - Luft. Es werden die bereits angesprochenen politischen Dimensionen der Atemluft sowie Technologien als Marker von Abhängigkeit und Unabhängigkeit tiefergehend erforscht.

Eine allgegenwärtige tödliche Erstickungsgefahr und die politische Dimension der Luft in Kunst und visueller Kultur bildet den Ausgangspunkt von Kerstin Borchhardts Beitrag zu Gas- und Atemmasken im 20. und 21. Jahrhundert. Sie fokussiert deren Ambivalenz als Symbol für lebensfeindliche Atmosphären, Dehumanisierung und Faschismus. Zudem skizziert sie die Bedeutung von Gasmasken für Fetische ebenso wie für künstlerische Institutionskritik am Beispiel der Street Art-Szene. Weitere politische Dimensionen eröffnen sich in den Überlegungen Borchhardts zur Bedeutung der Maske, die einerseits für Solidarität und andererseits für einen empfundenen Freiheitsverlust in der COVID-19-Pandemie steht.

Marcus Beckers Beitrag widmet sich ebenfalls Atemtechnologien - und zwar solchen, die im Vakuum notwendig sind. Der Autor untersucht die Weltraumfahrt in Science Fiction-Filmen unter dem Aspekt der Atmung als ästhetisches Problem und fokussiert dabei Raumanzug- 
Atem. Künste, Technologien und Architekturen der Moderne und Gegenwart

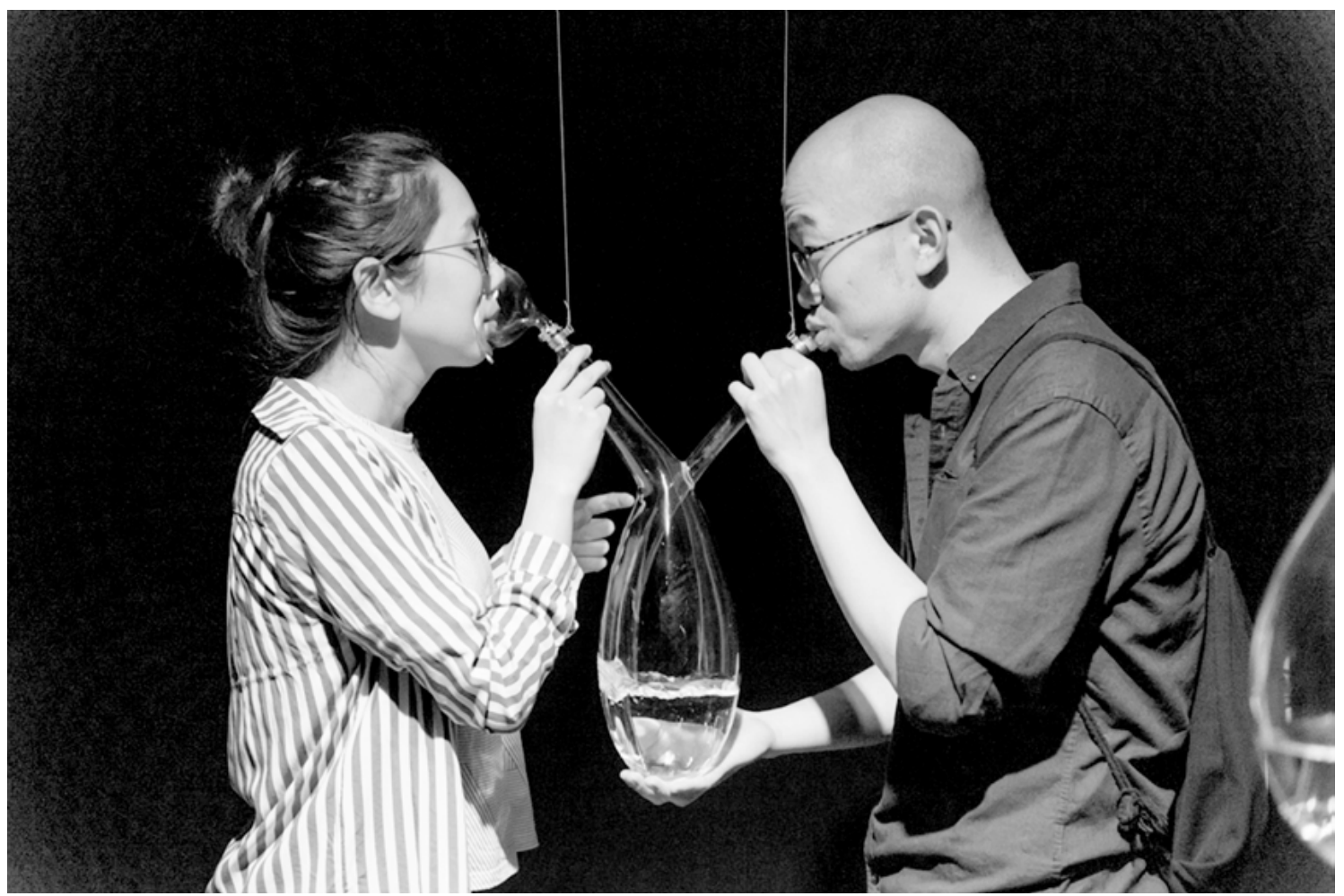


Design und dessen Bedeutung für die Filmdramaturgie, die audiovisuelle Gestaltung der Atmung in filmischen Krisensituationen sowie die Atmung als Pars pro Toto für Lebensfunktionen im Genre. ${ }^{77} \mathrm{Nicht}$ zuletzt thematisiert Becker den Niederschlag ökologischer Diskurse in jüngeren Produktionen wie Sunshine (2007), die spezifische Genderstereotype zitieren, welche sich in der Dichotomie von Sauerstoffgarten und Maschinen, Frau und Mann sowie in den Tätigkeiten des Gärtnerns und Messens offenbaren.

Die existentiell gefährdende Verwandlung des Lebensstoffes in toxisches $\mathrm{CO}_{2}$ hatte die berühmte Performance Breathing in - Breathing out (1977/1978) von Marina Abramović und Ulay vor Augen geführt. Sie rückt die Interdependenz zweier Liebender bis zu äußerster Erschöpfung und existentieller Gefahr in den Fokus. Edith Kollaths Partizipationswerk Liminal Passage (2018) erscheint fast wie eine Art therapeutische Antwort auf diese Performance. Sie basiert auf einem Glasobjekt mit einem Mund- und einem Atemteil (Abb. 6). Am Boden des runden Gefäßes befindet sich destilliertes Wasser. Die ausgeatmete Luft wird in das Wasser geleitet, dort von Viren und Bakterien gereinigt, und wird so von der Person am anderen Ende eingeatmet. Dadurch entsteht eine intime und dennoch vermittelte und distanzierte Kommunikation, ${ }^{78}$ ohne die Gefahr, mit potentiell ansteckenden Mikroorganismen aus der Mundflora des Gegenübers konfrontiert zu werden.

In der zeitgenössischen Kunst wird die künstliche Luftproduktion bzw. -manipulation ebenfalls mit Blick auf medizinische und ökologische Probleme zunehmend auf eine globale Ebene gehoben. Auf Konfrontation setzen die Pollution Pods (seit 2017) des Künstlers Michael Pinsky, die Annerose Keßler in ihrem Beitrag untersucht. Die Installation simuliert toxische Gase in globalen Industriemetropolen. Die Autorin eruiert das epistemische Potential der Kunst mit Blick auf das Spürbarmachen von globaler Umweltverschmutzung und deren desaströsen gesundheitlichen Folgen. Zugleich, und darin identifiziert Keßler die hier anvisierte aufklärerische Funktion dieser Kunstform, werden über die Immersion in die Lufträume hinaus auch Messwerte und weitere Informationen gegeben, womit Atemluft als Gegenstand wissenschaftlicher Forschungen und Kontrolle evident wird.

77 Siehe weiterführend zum Atem in Film und Kino Arthur Rose, „Breath in the technoscientific imaginary“, in: Rose A. Med Humanit 42, 2016, S. 31-35 und Quinlivan 2012 (wie Anm. 6).

78 Siehe Webseite der Künstlerin, verfügbar unter: http:// edithkollath.com/index.php/installations/liminal-passage (letzter Zugriff: 9.9.2020). 
Jedoch ist das Recht auf Atem und Luft keineswegs ein rein umweltpolitisches, sondern auch ein soziales und politisches Problem. Gloria Suttons Beitrag unternimmt vor dem Hintergrund des Rassismus in den USA eine Revision von Hans Haackes und Lygia Clarks frühen Werken. Die Autorin entwickelt ausgehend von Haackes Condensation Cube (1963-67) den rezeptionstheoretischen Ansatz der Komplizenschaft, die in der Teilhabe durch den Atem symbolisiert wird. Diese Komplizenschaft der Kunstrezipierenden stellt sie in einen Zusammenhang mit den rassistischen und kolonialen Strukturen, von welchen Kunstinstitutionen bis heute geprägt sind, und reflektiert ausgehend davon aktuelle Formen der Sozialkritik durch Kunstinstitutionen und Künstler*innen.

Ein Beispiel sind die Air Demonstrations des Künstlers Jammie Holmes angesichts der Ermordung George Floyds im Mai 2020. „I can't breathe" wurde bereits 2014 mit der Ermordung Eric Garners zu einem Schlüsselsatz der Protestkultur der Black Lives Matter-Bewegung (BLM), die - von Alicia Garza, Patrisse Cullors und Opal Tometi gegründet - sich gegen rassistische Polizeigewalt und rassistische Diskriminierung richtet. ${ }^{79}$ Auf diesen Satz haben auch Künstler*innen immer wieder rekurriert, um Haltung zu bekennen. ${ }^{80}$ Sutton reflektiert die Verletzlichkeit und ständige Bedrohung rassistisch diskriminierter Menschen, nicht zuletzt angesichts von Haackes kunstkritischem Verdikt: „No cop will be kept from shooting a black [sic] by all the lightenvironments in the world". 81

\section{Räume und Ökologien des Atems}

Tod und Leben, Bedrohung und Selbstverwirklichung sind zentrale Topoi, die als Extreme den verschiedenen künstlerischen und kulturellen Auseinandersetzungen mit dem Atem dialektisch anhängen. Die abschließende Sektion verdeutlicht erneut das utopische und schöpferische Potential, das der Arbeit mit Atemräumen in Moderne und Gegenwart zugeschrieben wurde und wird. In den Fokus rücken hier Ausstellungen und Architekturen als Atemräume, die als Räume des Lebens und des Austauschs ökologisch konzipiert sind.

Die Vorstellung der Ausstellung als Atemraum untersucht Elke Anna Werners Beitrag zu kuratorischen Konzepten Harald Szeemanns.

79 Zur Polizeigewalt in den USA sowie zur BLM-Bewegung und ihrer visuellen Kultur siehe exemplarisch Yates McKee, Strike Art. Contemporary Art and the PostOccupy Condition, London/New York 2016, insbes. S. 181-187 und S. 216-235.
80 Dazu siehe Henry Kaap, „,I Can't Breathe“ - Polizeigewalt und anti-rassistischer Protest in den USA“, in: Kritische Berichte 43, 2015, S. 86-95.

81 Haacke zit. n. Kaspar Koenig (Hg.), Hans Haacke: Framing and Being Framed: 7 Works 1970-75, Ausst.-Kat. New York 1975, S. 130. 
Ab Anfang der 1980er Jahre lässt sich diese Metapher des Atemraumes bei Szeemann hinsichtlich dramaturgischer, architektonischer, wirkungsästhetischer und semantischer Prinzipien seiner kuratorischen Praxis identifizieren. Daraus generierte er die Vorstellung einer Ausstellungsökologie. Bei Szeemann offenbart sich hier erneut ein dezidiert männliches Selbstbewusstsein, das eng mit dem entstehenden Selbstverständnis als freier Kurator in den 1980er Jahren verbunden ist. Die Affinität zu Diskursen rund um Vitalität und Ökologie ist zugleich auch den ,Lebensthemen' des Kurators und seinen Ausstellungsthemen geschuldet, die um zentrale Utopien der Kunst des 20. Jahrhunderts sowie die Verbindung von Kunst und Leben kreisen.

Spätestens seit der Moderne übten sich Künstler*innen darin, in Lufträume im Ausstellungs- oder Außenraum einzugreifen. Zu solchen Strategien gehören etwa der frühe Einsatz von Geruch in surrealistischen Ausstellungen Ende der 1930er Jahre..$^{82}$ Vito Acconci hingegen konzipierte mit Performances wie Seedbed (1972) den Ausstellungsraum selbst als atmenden Raum und entwickelte später ausgehend davon seine Architekturen des Windes, wie Rebecca Schönsee andernorts thematisiert. ${ }^{83}$ Andere Künstler*innen interessierten sich bereits verstärkt seit den 60er Jahren für klimatische Luftmanipulationen. Art \& Language (Michael Baldwin und Terry Atkinson) erdachten mit Air-Conditioning Show/Air Show (1966/67) eine Ausstellung mit leeren, neutralen Wänden, die konstant durch eine angenehme, weder zu warme, noch zu kalte Luft klimatisiert werden sollte. ${ }^{84}$ Künstlerische Kritik an der uniformierenden Air-Conditioning-Industrie wurde in der Kunst vermehrt seit den späten 1980ern geübt und durch Strategien der Irritation sowie durch die Erzeugung von Alteritätserfahrungen beispielsweise über Gerüche - thematisiert. ${ }^{85}$

Geruchsarchitekturen und Klimatisierung zwischen Standardisierung und Individualisierung von Architekturen bilden auch das Kernthema von Ole $W$. Fischers Beitrag. In diesem skizziert er eine neue Auffassung von Architektur von der Entwicklung moderner Klimaanlagen zu Beginn des 20. Jahrhunderts bis zu zeitgenössischen künstlerisch-architektonischen Konzeptionen. Fischer thematisiert, wie Architekturen individualisierte, nostalgische und utopische Atemräume über Gerüche erzeugen und deutet diese als Konzeptionen indi-

82 Caro Verbeek, „Surreale Aromen - Zur (Re-)Konstruktion des flüchtigen Erbes von Marcel Duchamp“, in: Ausst.-Kat. Basel 2015 (wie Anm. 10), S. 115-125.

83 Schönsee 2013 (wie Anm. 4), S. 273-275.

84 Michael Baldwin, „Remarks on Air-Conditioning“ (1967), in: Voids: A retrospective, Ausst.-Kat. Centre Pompidou/ Kunsthalle-Bern/Centre Pompidou-Metz, Zürich 2009, S. 65-69, hier: S. 67.
85 Dazu siehe Jim Drobnick, „Airchitecture: Guarded Breaths and the [cough] Art of Ventilation", in: Patrizia Di Bello/ Gabriel Koureas (Hg.), Art, History and the Senses. 1830 to the Present, Surrey/Burlington 2010, S. 147-166. Zu Margolles vgl. den Beitrag von Barbara Oettl in diesem Band. 
vidueller Klimakammern, die als Gegenbild eines homogenisierenden Globalismus zu lesen sind. Dabei müsste über die ideologische Verortung der untersuchten Positionen innerhalb der kritisierten Globalisierungsprozesse noch weiter reflektiert werden. Anhand von Fischers Auseinandersetzung mit Rahm und Terrain werden zudem die medizinischen und therapeutischen Aspekte zeitgenössischer architektonischatmosphärischer Entwürfe angesprochen - eine Tendenz, die ebenfalls von der Moderne des 20. Jahrhunderts geprägt worden ist. ${ }^{86}$

Die heutige sogenannte energieeffiziente Architektur schließt Luft ein, um Energie zu sparen und birgt dabei das Problem, die Luftqualität so zu verschlechtern, dass sie zu einem Gesundheitsrisiko wird. ${ }^{87}$ Entgegen dem Extrem einer hermetisch abgeschlossenen Architektur existierte bereits in der Moderne die Utopie flexibler, organischer Architekturen, die sich etwa an unterschiedliche Jahreszeiten anpassen können. Eine solche alternative Vorstellung von Architektur als Organismus mit einer atmenden ,Haut ${ }^{488}$ wird im abschließenden Gespräch zwischen Linn Burchert, Lorenzo Guiducci, Petra Gruber und Christiane Sauer diskutiert. Darin werden grundlegende Ideen und Ergebnisse des 2018 veranstalteten Berliner Workshop Breathing Skins sowie der Arbeit des Berliner Exzellenz-Clusters Matters of Activity. Image Space Material vorgestellt. Hier dienten pflanzliche Blätter als buchstäbliche Inspirationsquellen und Prototype für neue adaptive Architekturen, welche Wärme und Feuchtigkeit dynamisch regulieren. Dabei zeigte sich das Ideal der funktionalen Hautatmung, wie sie in der ersten Sektion ex negativo anhand der 1920er Jahre thematisiert wurde, als wesentliche Vorstellung für Architekturen der Zukunft.

\section{Zusammenfassung und Ausblick}

Die Perspektiven der Künste, Geisteswissenschaften sowie experimentellen wissenschaftlichen und gestalterischen Praktiken, die dieser Band versammelt, zeugen von den vielfältigen Dimensionen, Potentialen und Ängsten, die mit dem Atem in Moderne und Gegenwart verbunden sind: Atmung erscheint als physiologischer Prozess einerseits als planbar-regulierend, sogar als Zwang, andererseits aber als intuitiv, frei fließend und emanzipierend. Die Atmung dient künstlerischen und experimentellen Formgebungsprozessen ebenso wie sie deren Scheitern

86 Siehe weiterführend Imperfect Health: The Medicalization of Architecture, Ausst.-Kat. Canadian Centre for Architecture, Montreal 2012.

87 Porteous 2002 (wie Anm. 37), S. 52.

88 Ebd., S. 146-163. 
markiert, und dies in unterschiedlichsten Materialen - vom Pigment über den Webstoff zu Glas, Luftballon und Kaugummi. Das Ephemere des Atems zeigt sich insbesondere im Austausch von Umwelt und Organismus. Der Atem offenbart sich so als einerseits lebensspendend, andererseits als prekär und stets gefährdet bzw. gefährdend.

Im Fokus auf die humane Atmung, welche die Publikation größtenteils in ihr Zentrum stellt, lässt sich auch eine Einschränkung feststellen, die zwar in gewisser Weise symptomatisch für die mitunter kanonisierten und etablierten Kunst- und Kulturschaffenden, wissenschaftlichen Positionen und kulturellen Paradigmen in der untersuchten Zeitspanne sowie die fokussierten geographischen Räume ist, zugleich aber zunehmend hinterfragt wird. Die größtenteils anthropozentrischen Gegenstände und Zugänge in den Beiträgen sind Belege für die Abarbeitung an politischen und gesellschaftlichen Themen in Moderne und Gegenwart, die Atem als genuin menschlich bzw. Atem als gestalterische Potenz ausgehend vom Menschen denken und verhandeln. Das Atmen der Technologien, der Umwelt sowie die Atmung anderer Lebewesen und Stoffe scheint durchaus in einigen der Aufsätze auf - anhand von Blattatmung (Photosynthese), dem ,Ausatmen' des Wassers, der Erde und des Universums sowie Mensch-Technologie-Hybriden in posthumanen Zukunftsutopien und -dystopien. Der moderne Impetus des Künstlers als quasi-göttliche Macht, ,aufgeblasene“ Überhöhungen von Individualität sowie Versuche der Behauptung subjektiver Freiheit gegen Andere sind dem Atemthema kultur-, kunst-, bild- und architekturgeschichtlich jedoch in besonderem Maße eingeschrieben. Ebenso wie die Atmung zarte, liebevolle Verbindungen zu Anderen oder zur Welt ermöglichen kann, bricht sie sich gleichermaßen in diskriminierenden, gewalttätigen Impulsen der Abgrenzung und der Exklusion Bahn. 
